\title{
METAL MESH COUPLERS USING OPTICAL TUNNELING EFFECT AT MILLIMETER AND SUBMILLIMETER WAVELENGTHS
}

\author{
Jongsuck Bae ${ }^{\dagger}$, Jung-Chih Chiao ${ }^{\ddagger}, \mathrm{Koji} \mathrm{Mizuno}^{\dagger}$, and David B. Rutledge \\ ${ }^{\dagger}$ Research Institute of Electrical Communication, Tohoku University, \\ Katahira 2-1-1, Sendai 980, Japan \\ *Department of Electrical Engineering, California Institute of Technology \\ Pasadena, California 91125, USA
}

\begin{abstract}
A new metal mesh coupler that makes use of a tunneling effect of evanescent waves between a metal mesh and a dielectric plate, has been proposed as a quasi-optical component for millimeter and submillimeter wavelengths. Theoretical calculation and experimental measurement performed from $40 \mathrm{GHz}$ to $60 \mathrm{GHz}$ show that the transmittance of the coupler can be changed more than $50 \%$ for the variation of a spacing less than $0.18 \mathrm{~mm}$ between a capacitive mesh and a silicon plate at around $57 \mathrm{GHz}$.
\end{abstract}

\section{INTRODUCTION}

At millimeter and submillimeter wavelength regions, there has been substantial interest on the development and applications of quasi-optical devices, such as coherent power-combining [1] [2]. Fabry-Perot (FP) interferometers have been widely used as tunable optical couplers or filters as quasi-optical components. The FP-type optical couplers consist of two reflectors and make use of interference between propagation waves in the couplers to change their coupling coefficient. Consequently, the large and rapid change of the coupling coefficient with frequencies results in the narrow bandwidth of the FP-couplers. An optical tunneling type metal mesh (OTM) coupler has been proposed by us as a new quasi-optical component to overcome the tradeoff between bandwidth and coupling coefficient of the FP-couplers.

The OTM-coupler consists of an inductive or a capacitive metal mesh and a flat dielectric plate placed close to the surface of the mesh (Fig. 1), and makes use of an optical tunneling of evanescent waves to change reflectance and transmittance. The evanescent waves are induced by an incident wave on the mesh, and decay quickly away from the mesh, normally less than $\lambda / 20$ from the surface [3]. Therefore, in contrast to a FP-interferometer, the coupling coefficient of the OTM-coupler can be significantly changed by small adjustments of spacing between the mesh and the dielectric plate. In principle, the OTM-coupler can also be wide band because the transmission properties of the couplers depend primarily on the mesh parameters $(g, 2 a$, and $t$ illustrated in Fig. 1). In this paper, theoretical simulations and experimental results are reported to show the feasibility of the OTM-couplers.

\section{THEORETICAL ANALYSES}

The OTM-coupler consists of a silicon plate and a capacitive metal mesh on a quartz plate. The quartz plate has a refractive index of 2.12 and a thickness of $2 \mathrm{~mm}$. The silicon plate has a refractive index of 3.42 and a thickness of $1 \mathrm{~mm}$. The reflectance $\left(\left|S_{11}\right| 2\right)$ and transmittance $\left(\left|S_{21}\right| 2\right)$ of the OTM-couplers with different mesh parameters have been estimated by using the Hewlett-Packard High Frequency Structure Simulator (HFSS) and the Method of Moments (MOM) in the frequency range between $40 \mathrm{GHz}$ and $60 \mathrm{GHz}$.

In the first simulation, one-dimensional capacitive metal pattern (a strip grating) has been assumed because of its ease of calculation. The strips are assumed to be perfect conductors and have zero thickness. The $\mathrm{rf}$ loss of quartz and silicon plates are also assumed to be zero to simplify the calculations. A uniform plane wave is assumed to be normally incident on the grid, with the electric field polarized perpendicular to the strips. The sur-

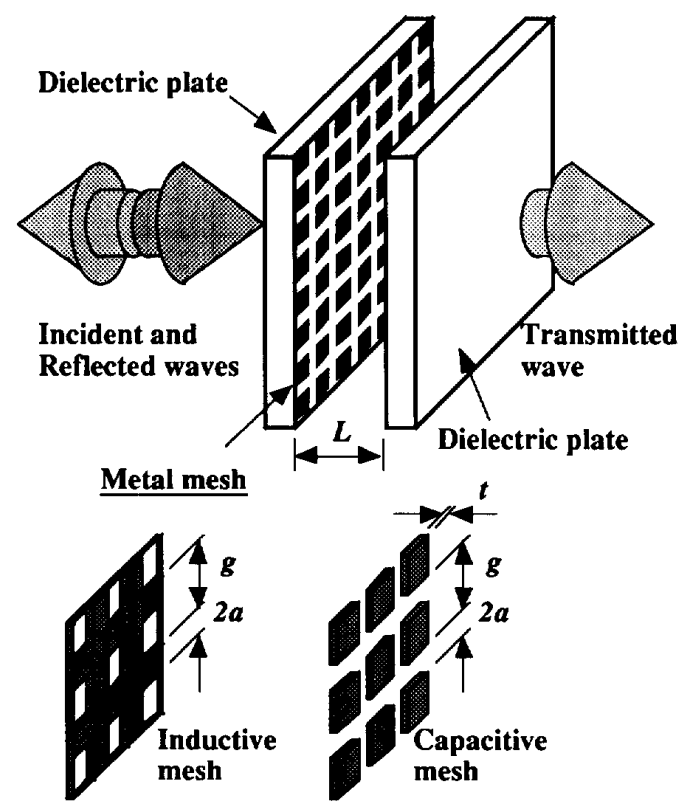

Fig. 1 Configuration of the OTM-coupler. 
face current distribution is determined using the method of moments [4]. Once the current distribution has been determined, the induced EMF technique was used to calculate the impedance of the capacitive grid. This technique is very similar to the one used by Eisenhart and Khan [5] and is further detailed by Weikle [6]. Finally, the impedance is used to find the reflected and transmitted wave through the structure using a simple transmission line circuit.

Fig. 2 shows the results calculated by both methods for reflectance and transmittance as a function of the spacing $L$ between the capacitive strip grating and the silicon plate at $60 \mathrm{GHz}$. The simulations have been done for the capacitive strip grating with a pitch $(g)$ of $1.465 \mathrm{~mm}$ and a gap $(2 a)$ of $0.585 \mathrm{~mm}$. The simulation results show that the optical tunneling effect clearly appears around $L=0$, and that the coupling coefficient can be varied more than a $50 \%$ range by changing $L$ only $0.18 \mathrm{~mm}$, i.e., $\lambda$ 28.

Fig. 3 shows the calculated transmittance of the OTM-coupler with a strip grating of $g=1.70 \mathrm{~mm}$ and $2 a=0.69 \mathrm{~mm}$ as a

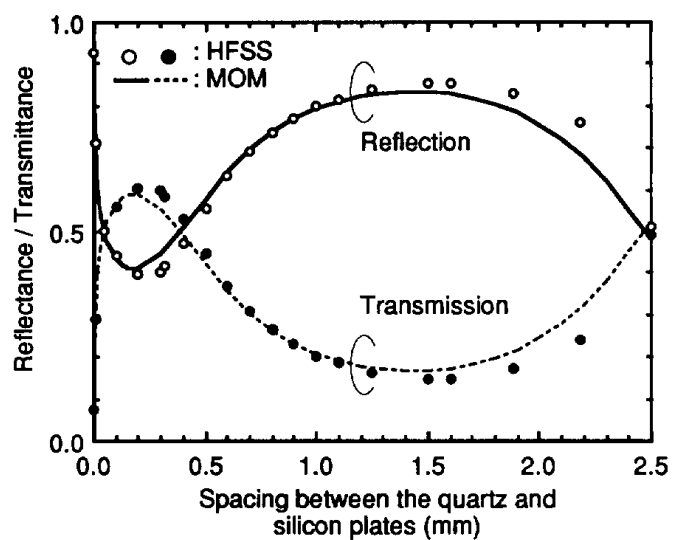

Fig. 2 Theoretical results for reflectance and transmittance at $60 \mathrm{GHz}$.

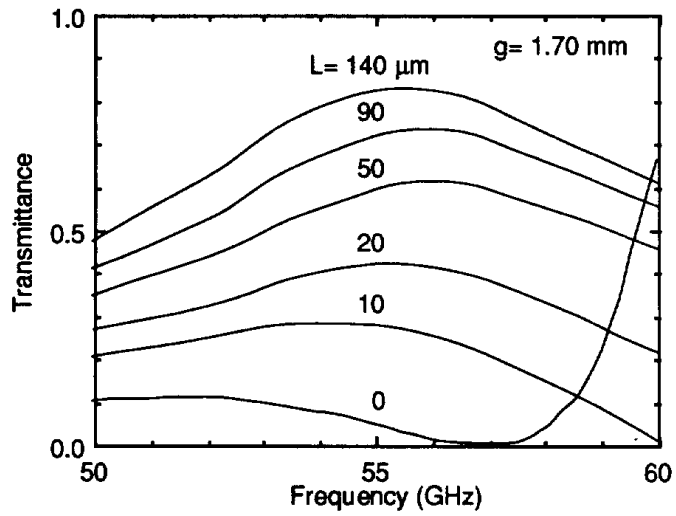

Fig. 3 Theoretical transmittance of the OTM-coupler with a mesh plate of pitch size $g$ of $1.70 \mathrm{~mm}$ as a function of frequency for different spacing $L$ between the mesh and silicon plate. function of frequency for different spacing of $L$. From the simulation results, it is seen that the OTM-coupler has a relatively flat band transmission property in the frequency range between 50 $\mathrm{GHz}$ and $57 \mathrm{GHz}$. The coupling coefficient can be changed by more than $40 \%$ over a $10 \%$ frequency range by varying $L$ within $0.14 \mathrm{~mm}$. The maximum change of $83 \%$ on the coupling coefficient can be achieved at $57 \mathrm{GHz}$. In Fig. 3, the transmittance of the coupler for $L=0$ is zero at $57 \mathrm{GHz}$ and increases abruptly when frequency is increased. This variation has been caused by a resonance effect in the strip grating [7].

\section{EXPERIMENTAL SETUP}

Experimental measurements have been done to confirm the theoretical predictions. The measurement setup includes an HP85106C millimeter-wave network analyzer, transmitting and receiving homs and an OTM-coupler as shown in Fig. 4. The frequency range is from $40 \mathrm{GHz}$ to $60 \mathrm{GHz}$. The spacing between the mesh and silicon plate is variable in the range form 0 to $10 \mathrm{~mm}$ with an accuracy of $\pm 10 \mu \mathrm{m}$. The parallelism between them is adjusted by using a He-Ne laser. The transmittances of the couplers were measured after calibration.

Three capacitive metal meshes with dimensions ( $g$, $2 a)=(1.48,0.59),(1.58,0.65)$ and $(1.70,0.69)$ in millimeter have been used in this experiment. Those meshes are fabricated on a z-cut quartz plate by using photolithographic techniques with an accuracy of $\pm 5 \mu \mathrm{m}$. The thickness of the metal meshes is about 1 $\mu \mathrm{m}$. The quartz plates are $40 \mathrm{~mm}$ in diameter and $2 \mathrm{~mm}$ in thickness, and the silicon plates are $63.5 \mathrm{~mm}$ in diameter and $1 \mathrm{~mm}$ in thickness.

The refractive indices of the quartz and silicon plates have been determined by measuring transmission properties using the same experimental setup as described above. From the measured results, the refractive index of the quartz plate is estimated to be $2.12 \pm 0.05$ and the field attenuation constant is about $0.5 \mathrm{Nep} /$ $\mathrm{cm}$. The refractive index of the silicon plate is $3.42 \pm 0.05$. A noticeable transmission loss of the silicon plate has not been observed in this frequency range.

\section{EXPERIMENTAL RESULTS}

Fig. 5 shows the theoretical and measured transmittances of the OTM-couplers with capacitive meshes with (a) $g=1.48$ $\mathrm{mm}$ and (b) $1.70 \mathrm{~mm}$ as a function of $L$ at frequencies of $40 \mathrm{GHz}$,

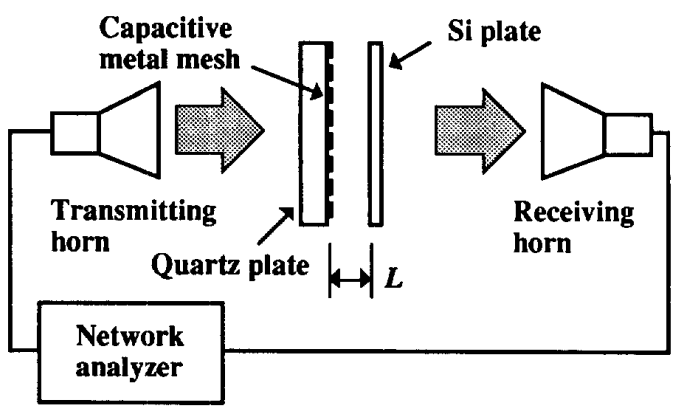

Fig. 4 Experimental setup. 
$50 \mathrm{GHz}$, and $58 \mathrm{GHz}$. The shapes of the curves are similar, however the theory is about $15 \%$ higher than the measurement at the peak values. From Fig. 5(b), it can be seen that the measured curves shift to right by the order of $10 \mu \mathrm{m}$ of $L$ in comparison with the theory. It might be caused by uncertainty of defining the origin position of $L$ in the experiment. The results show that the tunneling effect occurs only for the spacing less than $0.18 \mathrm{~mm}$. For larger spacing, the couplers behave like Fabry-Perot interferometers.

It is difficult to estimate an effective distance of tunneling precisely from Fig. 5 since the tunneling effect appears together with the Fabry-Perot etalon effect due to a finite thickness of the dielectric plate. Fig. 6 shows the measured transmittances with and without the tunneling effect for the OTM-coupler as a function of $L$ at $58 \mathrm{GHz}$ with the mesh size of $g=1.70 \mathrm{~mm}$. The circles indicate the same data shown in Fig. 5 (b) and solid line indicates measured transmittance for the same coupler, which was measured with an extra $\lambda / 2$ of $L$, shifted by $-\lambda / 2$ and superimposed on the circles in the same range of $L$. In Fig. 6 , the difference between two curves is caused by the tunneling effect alone. Comparing these two curves, it is found that the optical tunneling effect decays exponentially with a decay constant of about $60 \mathrm{Nep} /$
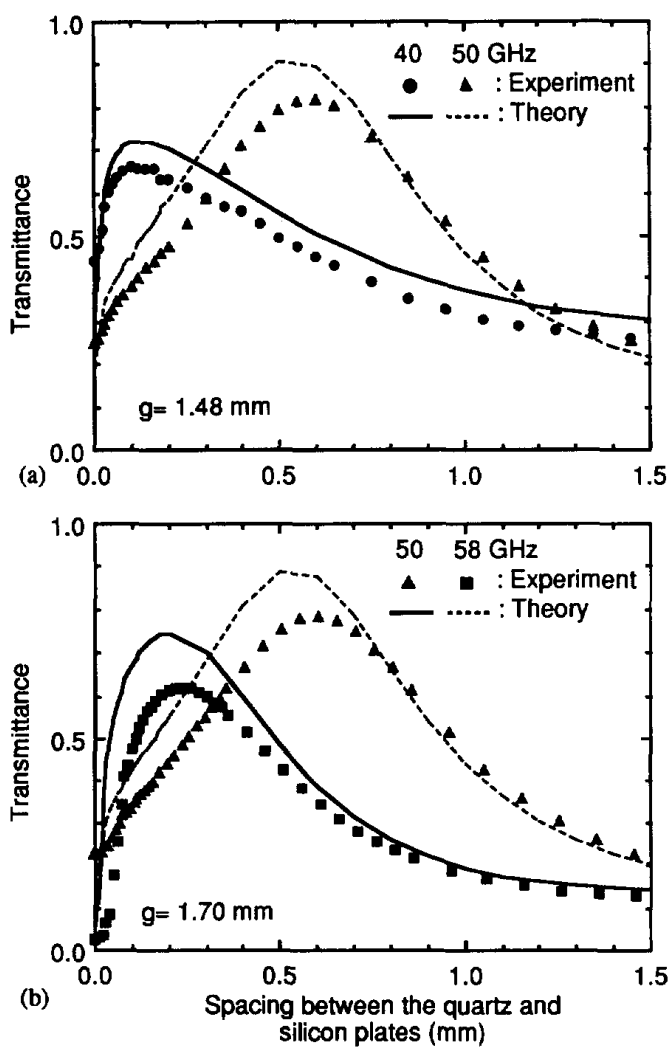

Fig. 5 Comparison of the theoretical and measured transmittances of the OTM-couplers with mesh plates of (a) $g=1.48 \mathrm{~mm}$ and (b) $g=1.70 \mathrm{~mm}$ at $40 \mathrm{GHz}, 50 \mathrm{GHz}$, and $58 \mathrm{GHz}$ $\mathrm{mm}$. The distance where the strength of the tunneling effect falls to $1 / \mathrm{e}^{2}$ of the maximum value is about $70 \mu \mathrm{m}$, i.e., $\lambda / 74$, including the shift of the origin position by $40 \mu \mathrm{m}$. For the other two OTM-couplers, similar values of the decay constant have been obtained at around $58 \mathrm{GHz}$. From Fourier optics theory [8] in the capacitive mesh, an evanescent wave of the first order has a decay constant of about $7 \mathrm{Nep} / \mathrm{mm}$. Thus, the estimated decay constant is more than 8 times larger than that of the evanescent wave induced on the metal mesh. This fact shows that the observed changes of transmittance in the coupler do not result from simple coupling between the evanescent field and the silicon plate.

Fig.7 compares the measured transmission properties of three OTM-couplers with $L=0$ as a function of frequency from $40 \mathrm{GHz}$ to $60 \mathrm{GHz}$. In the experiment, differences between the measured curves show that the changes of transmission property depend on the mesh parameters, mainly on the mesh pitch. The small fluctuations in Fig. 7 are caused by small amount of scattered wave from a mirror mount of the OTM-coupler. A strong dip and a peak of transmittance are observed from the experimental results. These drastic variation of transmittance indicates that the resonance exists, as predicted by the theory, at around 58 $\mathrm{GHz}$ for a mesh plate with $\mathrm{g}=1.70 \mathrm{~mm}$. In order to confirm the theory, the transmittance of the OTM-coupler with a capacitive mesh of $g=1.70 \mathrm{~mm}$ as a function of frequency from $50 \mathrm{GHz}$ to $60 \mathrm{GHz}$ for various values of $L$ has been measured and shown in Fig. 8. The predicted and measured transmittances are similar up to about $57 \mathrm{GHz}$ except a shift of about $50 \mu \mathrm{m}$ of $L$. The experimental results show that the coupling coefficient can be changed by more than $30 \%$ over a $10 \%$ frequency range by small variations of $L$. The change of coupling coefficient is smaller than the theoretical prediction by $10 \%$.

In the experiment as shown in Fig. 8, strong dips and peaks have been observed at around $58 \mathrm{GHz}$ for $L$ between 0 and 90 $\mu \mathrm{m}$. It is clear that there are two resonant frequencies. The strong peaks at higher resonant frequency $\left(f_{h}\right)$ around $59 \mathrm{GHz}$ are from Wood's anomaly [9], where the wavelength is equal to the period of the mesh multiplied the effective refractive index of the silicon plate. Since diffraction happens above $f_{h}$, an operation of the OTM-coupler is limited to the frequency of about $59 \mathrm{GHz}$. It

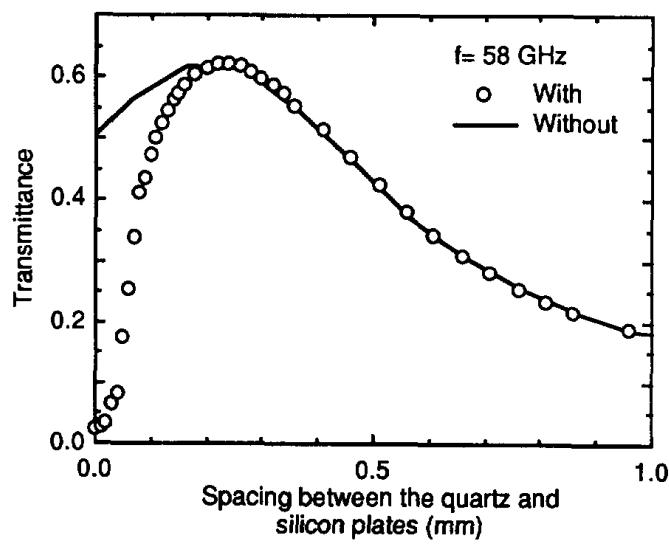

Fig. 6 Transmittance measured with and without a tunneling effect for the OTM-coupler with a mesh plate of $g=1.70 \mathrm{~mm}$. 
should be noted that this resonant frequency is higher than the frequency expected by the previous mesh theory $(51.6 \mathrm{GHz})$ [10].

From our experimental results, it is found that the effective refractive index of the silicon plate decreases as $L$ increases, approaching 3 as $L$ goes to 0 . This value is smaller than the actual refractive index of silicon, 3.42 . This effective refractive index has been also predicted by our theoretical simulation results. In Fig. 3, the two resonant frequencies predicted by the theory for $L=0$ are $57 \mathrm{GHz}$ and $60.7 \mathrm{GHz}$ while the experimental values are $57.6 \mathrm{GHz}$ and $59 \mathrm{GHz}$, respectively. When $L$ is larger than 10 $\mu \mathrm{m}$, the resonant frequencies obtained from the theory and the experiment are very different. In the experiment, the resonant effect in the OTM-coupler is very sensitive to the distance and parallelism between the mesh and the silicon plate. To explain the difference, more experiments at around resonant frequency will be required.

\section{CONCLUSION}

The optical tunneling type metal mesh (OTM) coupler has been proposed as a new quasi-optical component at millimeter and submillimeter wavelength regions. In the experimental results performed from $40 \mathrm{GHz}$ to $60 \mathrm{GHz}$, the coupling coefficient of the OTM-coupler can be changed by more than $30 \%$ over a $10 \%$ frequency range by adjusting a spacing of less than $\lambda / 28$ between the mesh and the silicon plate as predicted by the theory. The theoretical and experimental results have shown that the OTM- coupler is a potential component to be used at millimeter and submillimeter wavelengths.

\section{ACKNOWLEDGMENTS}

We would like to express our gratitude to Messrs. Y. Aburakawa, T. Fujii, and M. Miyajima at Tohoku University for fabricating the metal mesh and the other experimental apparatus. We also thank Michael DeLisio at Caletch for assistance with the theoretical simulations.

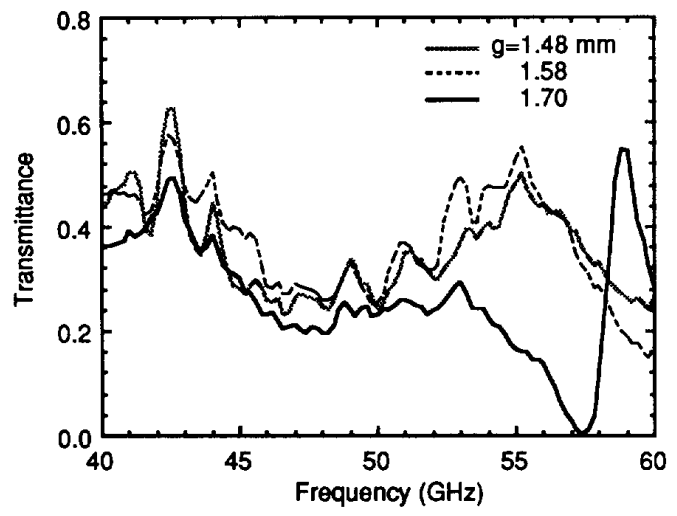

Fig. 7 Comparison of measured transmittances of the OTM-couplers with three different mesh sizes of $g=1.48,1.58$, and $1.70 \mathrm{~mm}$ as a function of frequency when $L=0$.

\section{REFERENCES}

[1] J. Bae, Y. Aburakawa, H. Kondo, T. Tanaka, and K. Mizuno, "Millimeter and Submillimeter Wave Quasi-Optical Oscillator with Gunn Diodes, “IEEE Trans. Microwave Theory Tech., vol. MTT-41, pp. 1851-1855, 1993.

[2] M. Kim, E. A. Sovero, J. B. Hacker, M.P. DeLisio, J. C Chiao, S. J. Li, D. R. Gagnon, J. J. Rosenberg, and D. B. Rutledge, "A 100-Element HBT Grid Amplifier," IEEE Trans. Microwave Theory Tech., vol. MTT-41, pp. 1762$1771,1993$.

[3] Z.S. Agronovich, V. A. Marchenko, and V. P. Shestopalov, "The Diffraction of Electromagnetic Waves from Plane Metallic Lattices," Sov. Phys. Tech. Phys., vol. 7, pp. 277 286, 1962.

[4] R. F. Harrington, "Field Computation by Moment Methods," Robert E. Krieger Publishing Company, Malabar, Florida, original ed. 1968, reprinted 1982.

[5] R. L. Eisenhart, P. J. Khan, "Theoretical and Experimental Analysis of a Waveguide Mounting Structure," IEEE Trans. Microwave Theory Tech., MTT-19, pp. 706-719, 1971.

[6] R. M. Weikle II, "Quasi-Optical Planar Grids for Microwave and Millimeter-Wave Power Combining," Ph.D. Dissertation, California Institute of Technology, 1992.

[7] M. S. Durschlag and T. A. DeTemple, "Far-IR Optical Properties of Freestanding and Dielectrically Backed Metal meshes," Appl. Opt., vol. 20, pp. 1245-1253, 1981.

[8] V. Yak., "Properties of a Fabry-Perot Interferometer with Mirrors in the Form of a Backed Metal Grid," So. Phys. Doklady, vol. 10, pp. 788-790, 1966.

[9] M. C. Hutley, Diffraction Gratings, London: Academic Press, 1982, pp. 175-210.

[10] R. C. Compton, L. B. Witbourn, and R. C. McPhedran, “ Strip grating at a dielectric interface and application of Babinet's principle," Appl. Opt., vol. 23, pp. 3236-3242, 1984.

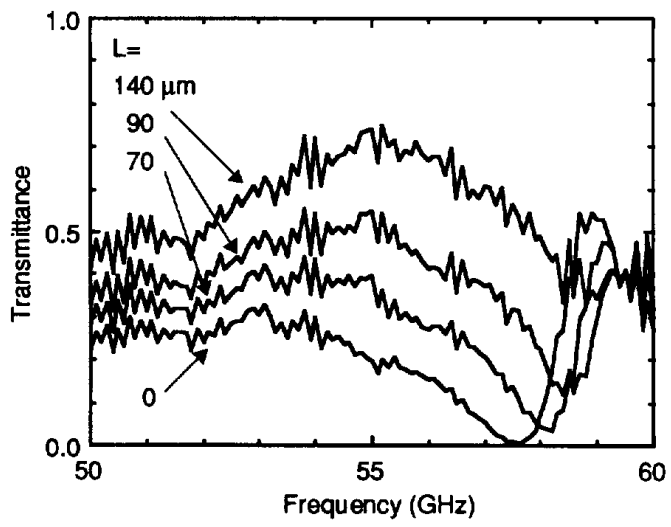

Fig. 8 Measured transmittance of the OTM-coupler with a mesh size of $g=1.70 \mathrm{~mm}$ as a function of frequency for different spacing $L$ between the mesh and silicon plate. 